\title{
Single fraction multimodal image guided focal salvage high-dose-rate brachytherapy for recurrent prostate cancer
}

\author{
Constantinos Zamboglou, MD 1.2, Hans-Christian Rischke, MD ', , Philipp Tobias Meyer, PhD, MD 2.3, \\ Sven Knobe, MSc2,4, Natalja Volgeova-Neher, MD1,2, Michael Kollefrath, MS 2,4, Cordula Annette Jilg, MD 2,5, \\ Prof. Anca Ligia Grosu, PhD, MD',2, Dimos Baltas, PhD, MSc 2,4, Malte Kroenig, MD2,5 \\ 'Department of Radiation Oncology, Medical Center - University of Freiburg, Faculty of Medicine, University of Freiburg, ${ }^{2}$ German Cancer \\ Consortium (DKTK). Partner Site Freiburg, ${ }^{3}$ Department of Nuclear Medicine, Medical Center - University of Freiburg, Faculty of Medicine, \\ University of Freiburg, ${ }^{4}$ Department of Radiation Oncology, Division of Medical Physics, Medical Center - University of Freiburg, Faculty of \\ Medicine, University of Freiburg, ${ }^{5}$ Department of Urology, Medical Center - University of Freiburg, Faculty of Medicine, University of Freiburg. \\ Germany
}

\begin{abstract}
Purpose: We present a novel method for treatment of locally recurrent prostate cancer (PCa) following radiation therapy: focal, multimodal image guided high-dose-rate (HDR) brachytherapy.

Material and methods: We treated two patients with recurrent PCa after primary (\#1) or adjuvant (\#2) external beam radiation therapy. Multiparametric magnetic resonance imaging (mpMRI), choline, positron emission tomography combined with computed tomography (PET/CT), or prostate-specific membrane antigen (PSMA)-PET combined with CT identified a single intraprostatic lesion. Positron emission tomography or magnetic resonance imaging - transrectal ultrasound (MRI-TRUS) fusion guided transperineal biopsy confirmed PCa within each target lesion. We defined a PET and mpMRI based gross tumor volume (GTV). A $5 \mathrm{~mm}$ isotropic margin was applied additionally to each lesion to generate a planning target volume (PTV), which accounts for technical fusion inaccuracies. A $\mathrm{D}_{90}$ of 18 Gy was intended in one fraction to each PTV using ultrasound guided HDR brachytherapy.

Results: Six month follow-up showed adequate prostate specific antygen (PSA) decline in both patients ( $\triangle$ PSA $83 \%$ in patient 1 and $\triangle$ PSA $59.3 \%$ in patient 2). Follow-up 3-tesla MRI revealed regressive disease in both patients and PSMA-PET/CT showed no evidence of active disease in patient \#1. No acute or late toxicities occurred.

Conclusions: Single fraction, focal, multimodal image guided salvage HDR brachytherapy for recurrent prostate cancer is a feasible therapy for selected patients with single lesions. This approach has to be evaluated in larger clinical trials.

J Contemp Brachytherapy 2016; 8, 3: 243-250

DOI: $10.5114 /$ jcb.2016.61067

Key words: HDR brachytherapy, multimodal imaging, recurrent prostate cancer, salvage therapy.

\section{Purpose}

Biochemical recurrence (BCR) after primary or adjuvant radiotherapy $(\mathrm{RT})$ for prostate cancer $(\mathrm{PCa})$ remains still a significant problem. Recurrence rates between $15-20 \%$ in low-risk patients and up to $40 \%$ in high-risk patients after primary low-dose-rate brachytherapy (LDR-BT) or primary intensity modulated radiotherapy (IMRT) are reported [1,2]. Recently, Stone et al. showed that recurrence rate after primary LDR-BT is dose dependent [3]. Zelefsky et al. showed that $15-20 \%$ of patients treated with dose escalated external beam RT have residual disease [4].

Rising prostate specific antigen (PSA) serum level suggests recurrent disease but fails to specify the localization. Multiparametric magnetic resonance imaging (mpMRI) [5], choline, or prostate specific membrane antigen (PSMA) positron emission tomography combined with computed tomography (PET/CT) [6,7], and MR-lymphangiography [8] can identify local recurrent PCa. Targeted biopsies should be performed for validating the imaging based target lesions. Especially mpMRI-guided biopsies revealed promising results in detection of recurrent $\mathrm{PCa}$ after RT, showing higher detection rates of significant PCa compared to randomly assigned biopsies $[9,10]$.
\end{abstract}


The aim of salvage RT is local PCa control and minimal side effects [11]. Mortality is decreased in patients receiving salvage therapy $(7 \%)$, whereas $25 \%$ of patients die without salvage therapy [12]. Prognostic factors, such as PSA doubling time, initial T-status, Gleason score, patient life expectancy, urinary function, and disease free interval, serve as selecting criteria for salvage therapy $[13,14]$. Several local treatment modalities have been evaluated as potentially curative options for localized recurrences: cryotherapy [15], radical prostatectomy [16], high-intensity focused ultrasound (HIFU) [17], stereotactic RT [18], and LDR-BT or high-dose-rate BT (HDR-BT) $[19,20,21]$.

Even though long biochemical free survival intervals have been reported for salvage BT, increased toxicity has been detected as well in the salvage setting when compared to primary therapy $[22,23]$. Thus, the concept of partial gland or focal BT on recurrent PCa evolved. First studies showed promising results using focal salvage LDR-BT [24,25]. Target volume for dose delivery was defined by combining mpMRI and transrectal ultrasonography (TRUS) biopsies, or by combining transperineal TRUS biopsies and low dose areas of previous RT. Recently, Yamada et al. supposed 3D-mapping based on previously performed mpMRI-guided transperineal TRUS biopsies as a guidance tool for focal LDR-BT on recurrent PCa [22].

This work presents a new method for salvage therapy in only local recurrent PCa: single fraction HDR-BT for multimodal image defined recurrent $\mathrm{PCa}$.

\section{Material and methods}

\section{Patient selection}

Two patients were presented with biochemical failure according to Phoenix criteria [26]. The patients fulfilled following criteria: functional (mpMRI) and molecular imaging $\left({ }^{18} \mathrm{~F}\right.$-choline PET/CT or PSMA PET/CT, using the ligand ${ }^{68}$ Ga-HBED-CC-PSMA [27]), revealed a single lesion without metastatic disease, ECOG performance status 0 , rectoscopy showed no/mild proctitis, no urethral constriction, and external beam radiation therapy was over 5 years ago. The patients has given a written consent prior to any intervention.

\section{Targeted transperineal transrectal ultrasonography biopsy}

One patient underwent MRI-guided transperineal TRUS biopsy, and in one patient choline PET/CT guided transperineal TRUS biopsy was performed. Gross tumor volumes for targeted biopsies were delineated according to Zamboglou et al. [28] in iPlan (iPLan RT Image 4.1.2., Brainlab, Germany). The iPlan software contains a SUV-thresholding tool, which was mandatory for delineation of GTV-PET according to our clinical protocol. Possible co-registration uncertainties between CT or MRI and TRUS images were accounted for by GTV enlargement by a $5 \mathrm{~mm}$ isotropic margin to create the planning target volume (PTV). The T2w MRI or CT images with delineated GTVs and PTVs were transferred via DICOM to Oncentra Prostate intraoperative real-time planning system $(\mathrm{OcP}$,
Version 4.2.21, Elekta Brachytherapy, The Netherlands). The prostate gland and the rectum were delineated in OcP by an experienced radiation oncologist.

All biopsies were performed in general anesthesia and in lithotomy position. A TRUS biplane probe (Pro Focus Ultra View 2202, BK Medical, Denmark), which is registered to a template biopsy system and the OcP was placed transrectally. Using a stepper device with electronic tracking of rotation and translation, a 3D image set of the prostate was generated in OcP. Computed tomography or MRI (T2w) data with defined GTVs and PTVs were manually registered to TRUS data by urologist and radiation oncologist in consensus. For alignment of the prostatic gland in TRUS and CT/MR images, the delineated contours of rectum and prostate (patient 1 ) or tumoral mass (patient 2) served as orientation in 3 dimensions. In the first step, the contoured rectum in CT/MRI was used for alignment with the TRUS images in the Z-axis. The contoured prostatic gland/tumoral mass guided further alignment between MRI/CT and TRUS in the X-and $Y$-axis. Rotations in all 3 dimensions were implemented in the final registration step. No scaling of the prostate/tumoral mass in MRI/CT or TRUS was performed during registration process. Subsequently, needle positioning for targeted and systematic biopsies was planned. A standard HDR-BT template (GfM, Germany) was used. The needle positions were $2.5 \mathrm{~mm}$ apart. Two brachytherapy needles were inserted to stabilize the position of prostate during biopsy. To exclude a shift of prostate/tumoral mass during preplanning procedure, visual re-assessment of registration between CT/MRI and TRUS was performed, taking into account the contours of rectum and prostate/tumoral mass. Insertion and positioning of biopsy needles was done under live sagittal TRUS imaging until correct positions were reached. Biopsy cores were labelled and processed according to routine procedure at our clinic.

\section{Focal high-dose-rate brachytherapy}

For HDR-BT, the same technique as for targeted transperineal biopsies was used. Multimodal image co-registration, GTV delineation, and expansion to PTVs were done in iPlan. Subsequently, the preplanning, transperineal implantation of the steel needles $(1.5 \mathrm{~mm}$ diameter) using live TRUS guidance, and intraoperative treatment planning were performed in OcP [29]. Five (patient 1) and seven (patient 2) brachytherapy needles, respectively, were inserted to cover the entire PTV using an ${ }^{192}$ Ir HDR afterloading system (microSelectron-HDR, Digital Version 3, Elekta Brachytherapy, The Netherlands). Brachytherapy was performed with an aimed prescription dose of $18 \mathrm{~Gy}$, according to [30,31]. The EQD2 equieffective dose [32] of a 18 Gy single fraction based on the AAPM guidelines [33] is 75.6 Gy using an $\alpha / \beta$ value of $3.0 \mathrm{~Gy}$. Individually planned $D_{90}$ was $16.4 \mathrm{~Gy}$ and $23.7 \mathrm{~Gy}$, respectively (91\% and $131 \%$ of aimed prescription dose) (Table 1). Dose restrictions for rectum and urethra were modified to Yamada et al. [21], where in maximum $120 \%$ and $100 \%$ of reference prescription dose was tolerated to the urethra and rectum, respective- 
Table 1. Dose characteristics

\begin{tabular}{|c|c|c|c|c|c|c|c|c|c|}
\hline & $\begin{array}{c}\text { PTV } \\
\text { volume } \\
\left(\mathrm{cm}^{3}\right)\end{array}$ & $\begin{array}{c}\text { Aimed } \\
\text { prescription } \\
\text { dose (Gy) }\end{array}$ & $\begin{array}{l}\text { PTV D } \\
\text { (Gy) }\end{array}$ & $\begin{array}{c}\text { PTV } \\
V_{100}\left(\mathrm{~cm}^{3}\right)\end{array}$ & $\begin{array}{l}\text { Urethra } \\
D_{10}(G y)\end{array}$ & $\begin{array}{c}\text { Urethra } \\
D_{0.1 c c}(G y)\end{array}$ & $\begin{array}{l}\text { Rectum } \\
\mathrm{D}_{2 \mathrm{cc}}(\mathrm{Gy})\end{array}$ & $\begin{array}{c}\text { Rectum } \\
D_{0.1 c c}(G y)\end{array}$ & $\begin{array}{c}\text { Bladder } \\
\mathrm{D}_{0.1 c c}(\mathrm{~Gy})\end{array}$ \\
\hline Patient 1 & 5.63 & 18.00 & 16.39 & 4.65 & 11.33 & 5.07 & 3.22 & 10.60 & $N / A$ \\
\hline Patient 2 & 1.16 & 18.00 & 23.67 & 1.14 & 10 & 8.71 & 4.04 & 10.70 & 4.60 \\
\hline
\end{tabular}

PTV - planning target volume, $D_{90}$ - the minimum dose received by $90 \%$ of the prostate volume, $V_{100}$ - the percent volume of the post-implant prostate receiving $100 \%$ of the prescribed dose, Urethra $D_{10}$ - the minimum doses received by $10 \%$ of the urethral volume, Urethra $D_{0.1 c c}$ - the minimum dose received by $0.1 \mathrm{cc}$ of the urethra, Rectum $D_{2 c c}$ - the minimum dose received by $2 c c$ of the rectum, $R D_{0.1 c c}$ - the minimum dose received by $0.1 \mathrm{cc}$ of the rectum, $B l a d d e r D_{0.1 c c}-$ the minimum dose received by 0.1 cc of the bladder

Table 2. Inverse planning parameters. Dose-volume histogram-based optimization (HIPO) algorithm was used in OcP. The aimed presumptive dose was 18 Gy (100\%)

\begin{tabular}{lcccc} 
Volume of interest & Type & Low-dose limit (Gy) & Important factor & High-dose limit (Gy) \\
\hline PTV_MRI + PET & Target & $100 \%(18 \mathrm{~Gy})$ & 8 & $150 \%(27 \mathrm{~Gy})$ \\
\hline Urethra_US & OAR & - & - & $120 \%(21.6 \mathrm{~Gy})$ \\
\hline Rectum_US & OAR & - & - & $75 \%(13.5 \mathrm{~Gy})$
\end{tabular}

PTV - planning target volume, MRI - magnetic resonance imaging, PET - positron emission tomography, US - ultrasonography, OAR - organ at risk

ly. Dose-volume histogram-based optimization (DVHO) inverse planning was used in OcP (Table 2).

\section{Case reports}

\section{Patient 1}

In May 2008, a 70 year old patient was presented with histological confirmed PCa (Gleason score 6), TNM-status pT1b cNx cMx (negative bone scan), and serum PSA level $9.39 \mathrm{ng} / \mathrm{ml}$ for primary RT. From July till September 2008, 74 Gy in 2 Gy fractions were delivered. In March 2014, the patient presented PSA serum level of $4.14 \mathrm{ng} /$ $\mathrm{ml} .{ }^{18} \mathrm{~F}$-choline PET/CT and mpMRI revealed PCa in the right prostatic lobe, no metastatic disease was detected. Between March and August 2014, 2 trans-rectal biopsies failed to detect PCa. Stable disease occurred in a second choline PET/CT examination in December 2014. Choline PET/CT guided biopsy was performed in February 2015.
Histological analysis revealed PCa (Gleason score 8) in 4 of 9 targeted-biopsy cores, whereas in 10 randomly assigned probes no PCa was described. In April 2015, multiparametric 3 tesla-MRI depicted only the histological confirmed lesion. Androgen deprivation therapy was offered but patient refused due to possible toxicity.

Before HDR-BT, an experienced nuclear physician, radiologist, and radiation oncologist delineated gross tumor volume (GTV) based on choline PET (GTV-PET) and mpMRI (GTV-MRI) in consensus. Both GTVs were enlarged by applying $5 \mathrm{~mm}$ isotropic margins to create planning target volume (PTV) in iPlan (Figure 1). All single PTVs were then merged to a single PTV (volume $5.62 \mathrm{~cm}^{3}$ ). In April 2015, the patient presented serum PSA level of $5.65 \mathrm{ng} / \mathrm{ml}$ for focal HDR-BT. A D90 of 16.4 Gy was planned in one fraction (Figure 2). In June 2015, the patient was presented for first follow-up with PSA serum-level $0.67 \mathrm{ng} / \mathrm{ml}$. According to RTOG late radiation
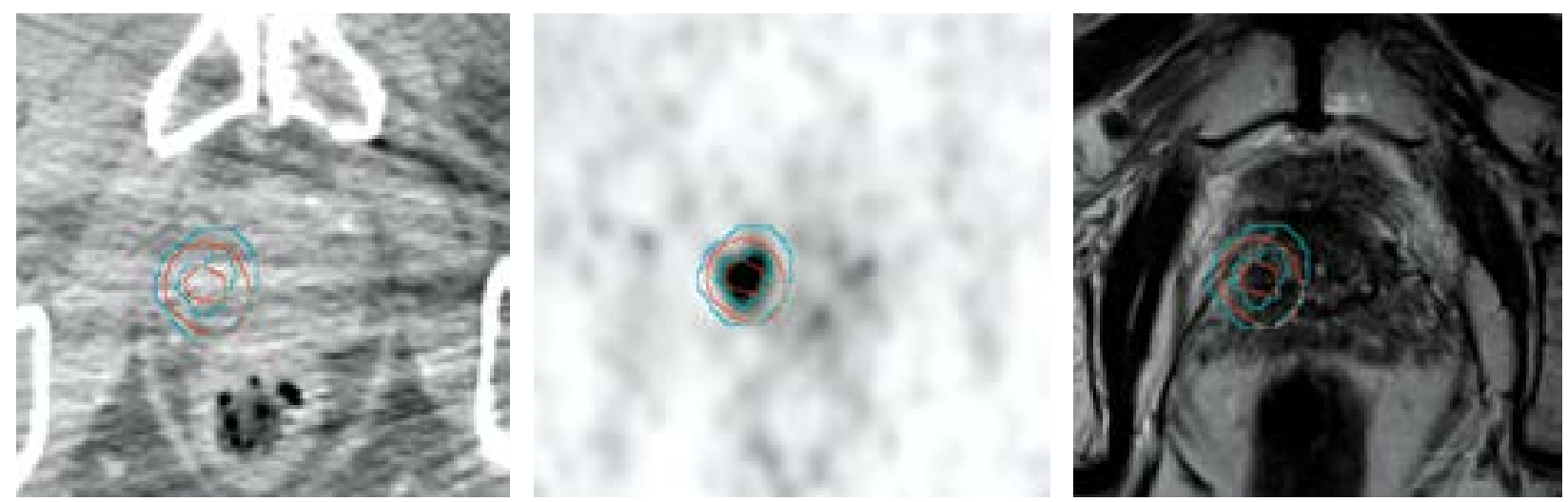

Fig. 1. Patient 1. Left: axial computed tomography (CT)-slice of choline positron emission tomography combined with computed tomography (PET/CT), middle: axial choline PET slice, right: T2w 3 tesla magnetic resonance imaging (MRI)-slice (raw data). Isotropic enlargement with $5 \mathrm{~mm}$ of gross tumor volumes (GTVs) revealed planning target volumes (PTVs). GTV-/PTV-MRI (red) and GTV-/PTV-PET (blue) revealed high spatial overlap. Merging of both PTVs leaded to a multimodal imaging PTV, which served as the target for focal high-dose-rate brachytherapy 

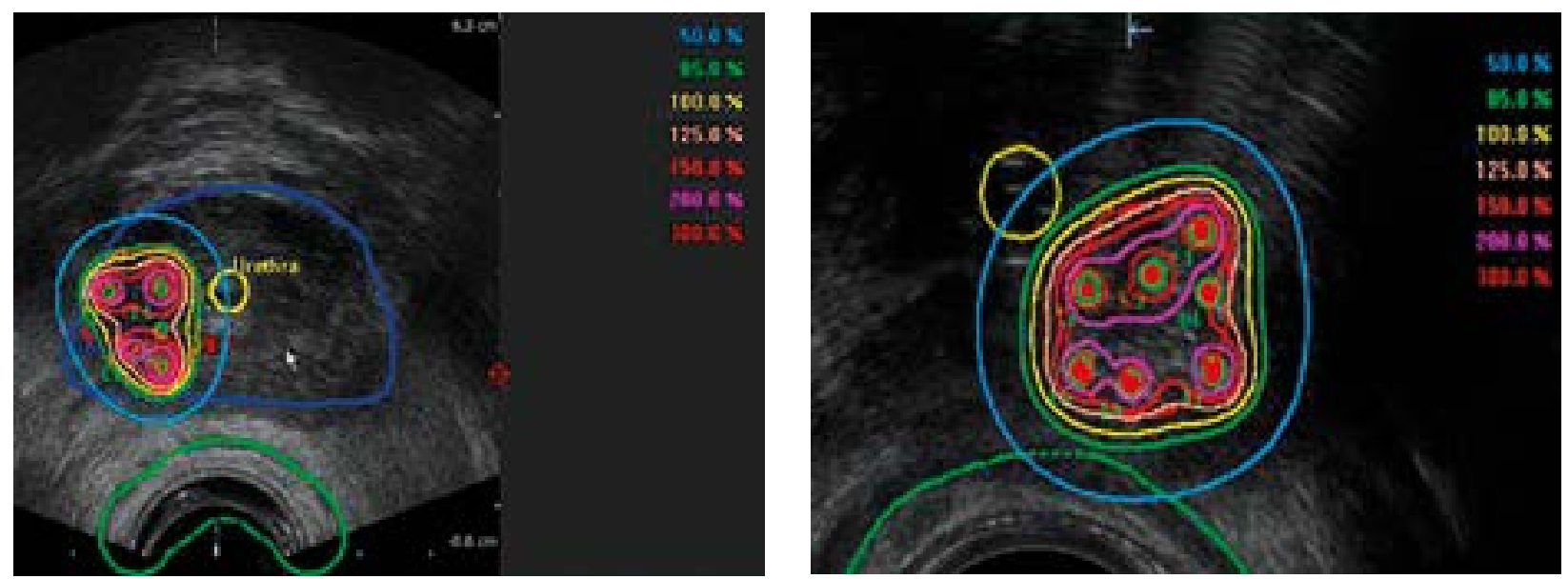

Fig. 2. Transrectal ultrasound (TRUS) images with isodoses. Transversal TRUS images with isodoses (patient 1, left and patient 2 ,

right). Reference prescription dose for both planning target volumes (PTVs) was 18 Gy (100\%)

morbidity score, no GI and no GU toxicity occurred. Erectile function was normal. 3 Tesla mpMRI revealed regressive disease (Figure 3). In September 2015 and February 2016, second and third follow-up examinations revealed a stable PSA-level of $0.67 \mathrm{ng} / \mathrm{ml}$ and no toxicity occurred. A PSMA PET/CT scan showed a complete remission in April 2016.

\section{Patient 2}

In July 2006, a 59 year old patient was presented for primary treatment. Radical prostatectomy was performed for primary $\mathrm{PCa}$ (pT3b cN0 cM0, Gleason score 9). Subsequently, androgen deprivation therapy with flutamide and LHRH analogues was given. From September till November 2006, adjuvant external beam radiation therapy was performed, delivering $64 \mathrm{~Gy}$ in $2 \mathrm{~Gy}$. In May 2015, the patient had PSA $3.07 \mathrm{ng} / \mathrm{ml}$. PSMA PET/ $\mathrm{CT}$ and mpMRI depicted a tumoral mass with high spatial conformity, suspected for recurrent PCa (Figure 4). GTV-delineation for biopsy and for focal HDR-BT was based on PET and MRI images. MpMRI-guided transperineal biopsy was performed in November 2015 with 4 from 4 targeted biopsy cores positive for PCa (Gleason score was not determinable). Using single-fraction focal HDR-BT, a D90 of 23.7 Gy was planned to the PTV (GTV $+5 \mathrm{~mm}, 1.16 \mathrm{~cm}^{3}$ ) (Figure 2). No acute toxicity occurred. In January 2016, the patient presented for first follow-up with PSA serum-level $1.25 \mathrm{ng} / \mathrm{ml}$, which was stable in March 2016. No GI and no GU toxicity was reported. Erectile function was constantly restricted after prostatectomy.

\section{Discussion}

Patients with locoregional recurrent PCa after external RT may be cured. Thus, the need for a precise and secure salvage treatment option has gained interest in the past years. In this work, we present a new method for treatment of locally recurrent PCa: single dose HDR-BT for multimodal guided and targeted biopsy confirmed PCa. MpMRI and ${ }^{18}$ F-choline PET/CT or PSMA PET/CT depicted a single lesion of recurrent PC in each patient. MRI/PET guided transperineal biopsy confirmed the single lesion. The prescription dose defined as $\mathrm{D}_{90}$ of $18 \mathrm{~Gy}$ was planned in 16.4 and 23.7 Gy to multimodal (PET and MRI) delineated GTVs using HDR-BT in one fraction. No biochemical failure occurred and treatment was well tolerated.

We would like to address further possible improvement of our presented methodology. Molecular imaging was performed using choline PET/CT (patient 1) or PSMA PET/CT (patient 2). Cimitan et al. reported that the detection rate for ${ }^{18} \mathrm{C}$-choline-PET/CT in patients with recurrent PCa and Gleason score $>7$ was $91 \%$, when PSA values were $>2 \mathrm{ng} / \mathrm{ml}$. However, Grosu et al., proved that choline PET/CT failed to distinguish PCa from non-tumoral tissue in most of the patients with primary PCa using PET with histology co-registration [34]. Furthermore, several groups could show that PSMA PET/ CT may offer superior performance in detection of recurrent PCa compared to choline PET/CT $[35,36]$. Thus, we suggest using PSMA PET/CT instead of choline PET/CT for target delineation. Although we used a well-established RT-planning software (iPlan), inaccuracy in co-registration between PET, CT, and mpMRI images occurred. It is well known that the prostate undergoes constantly changes in size (up to $10 \%$ ) and location (up to $5 \mathrm{~mm}$ ) due to bowel and bladder movement [37]. Thus, co-registration between CT and PET or MRI can be impeded due to different acquisition times between the imaging modalities. Additionally, CT images suffer from lower soft tissue contrast compared to MRI. This leads to reduced depiction of the prostatic gland, especially in the apex due to its close proximity to the pelvic floor muscles $[38,39]$. This may lead to decreased performance of manual and software based co-registration between MRI and CT images of the prostatic gland. The usage of integrated PET/MRI-systems may decrease this uncertainty [40]. Additionally, co-registration between MRI/CT and TRUS images for biopsy and for HDR-BT was performed manually by visual control. Radtke et al. shows promising results in image fusion between MR and TRUS images 
$\mathrm{T} 2 \mathrm{~W}$

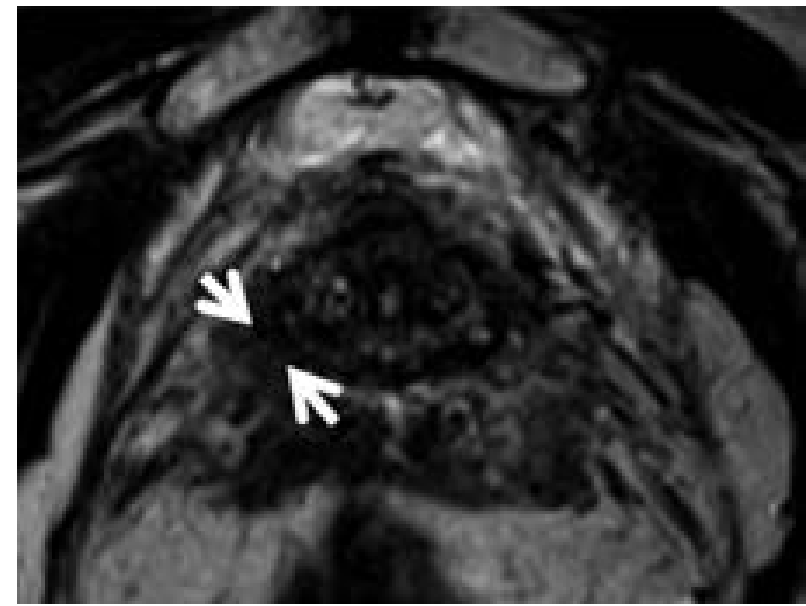

DWI

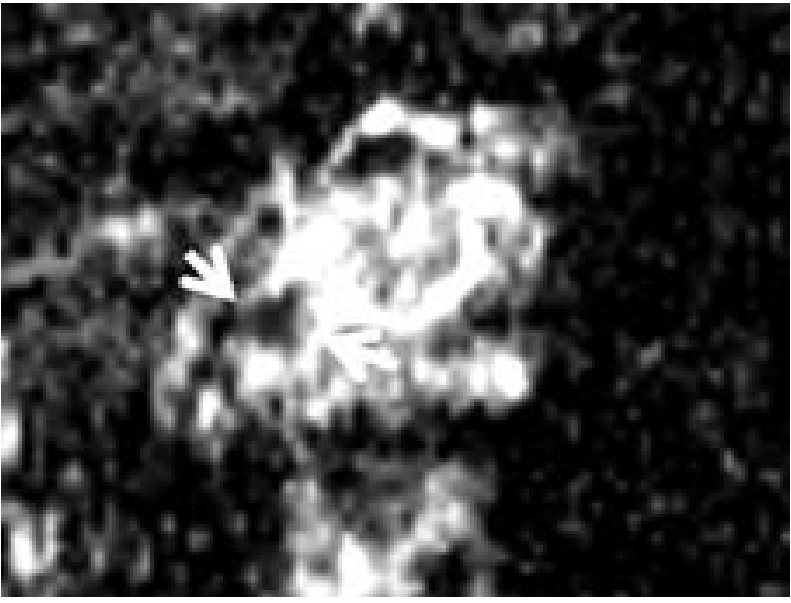

DCE

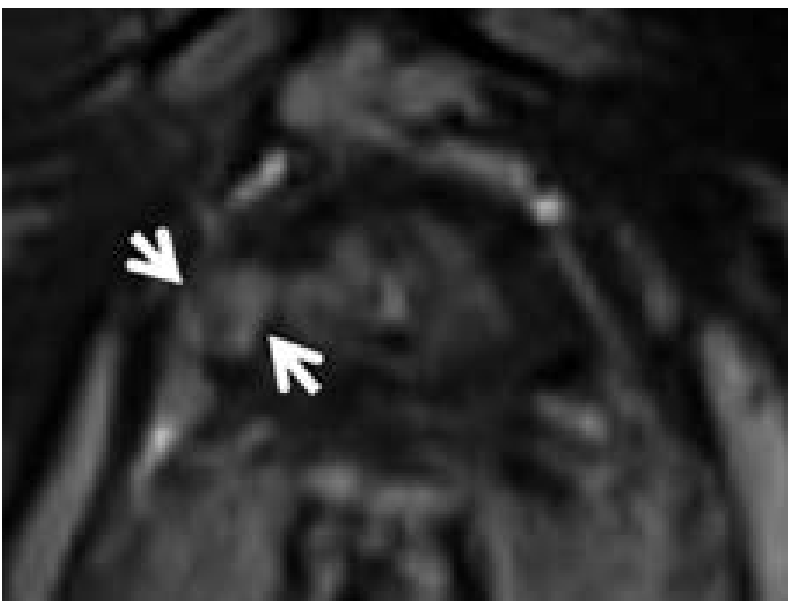

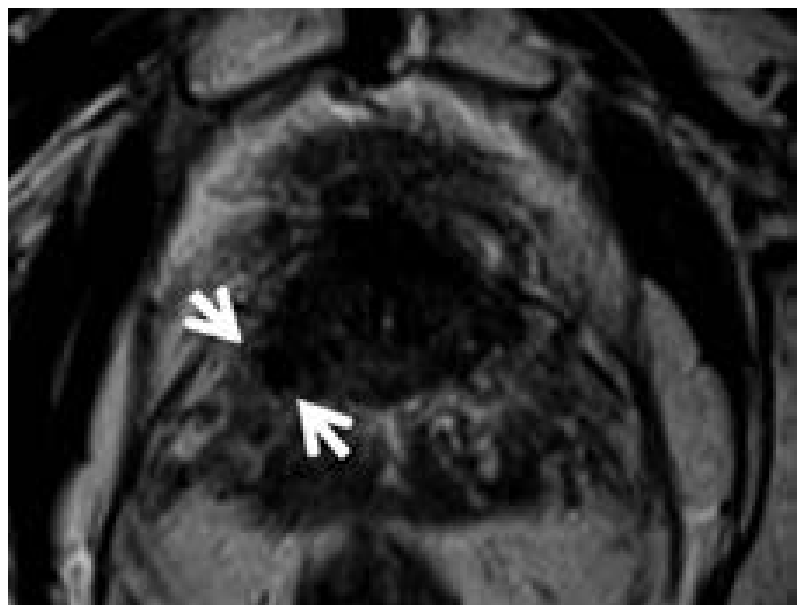
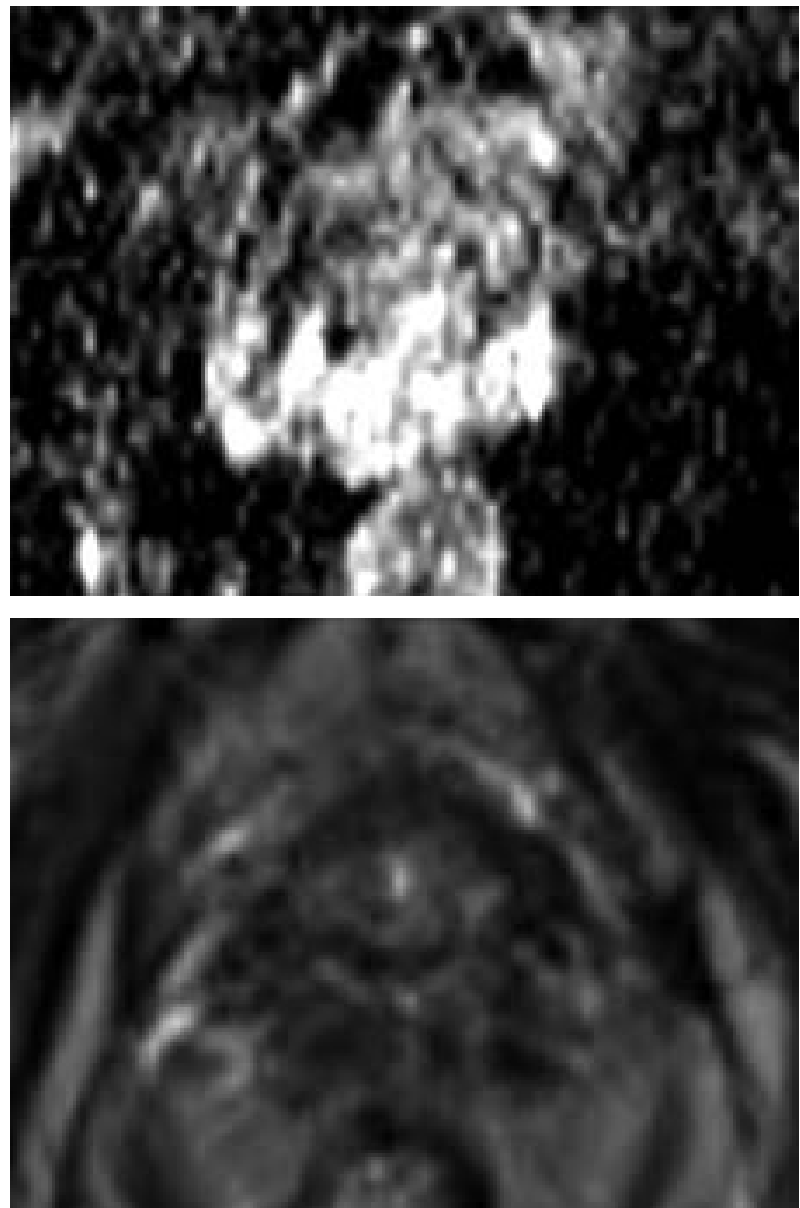

Fig. 3. Follow-up multiparametric magnetic resonance imaging (mpMRI). MpMRI before (left) and 2 months after (right) focal high-dose-rate brachytherapy in patient 1. T2w images: after HDR-BT recurrent prostate cancer (PCa) was stable in size but demarcation increased (white arrows). Diffusion weighted imaging (DWI) (ADC-map): before brachytherapy (BT) diffusion restriction was observed (white arrows), which decreased after BT. dynamic contrast-enhanced (DCE) imaging: pre-BT contrast enhancement (white arrows) diminished after BT

using non-rigid, elastical registration for MRI-targeted biopsies [41]. Typical changes in prostatic shape are caused mechanically by the TRUS probe insertion in the rectum and the pressure required for assuring an adequate acoustic coupling for the TRUS imaging. In performing mpMRI and PET/CT in our study, no endorectal coil was used. Thus, during pre-therapeutical imaging, no mechanical deformation of the prostate occurred. A transrectal dummy may be used to simulate the TRUS probe during MRI or PET/CT scans [42]. In our study, we conducted a MRI- and PET-guided TRUS biopsy before $\mathrm{BT}$. The usage of a 3D-documented biopsy mapping tech- 

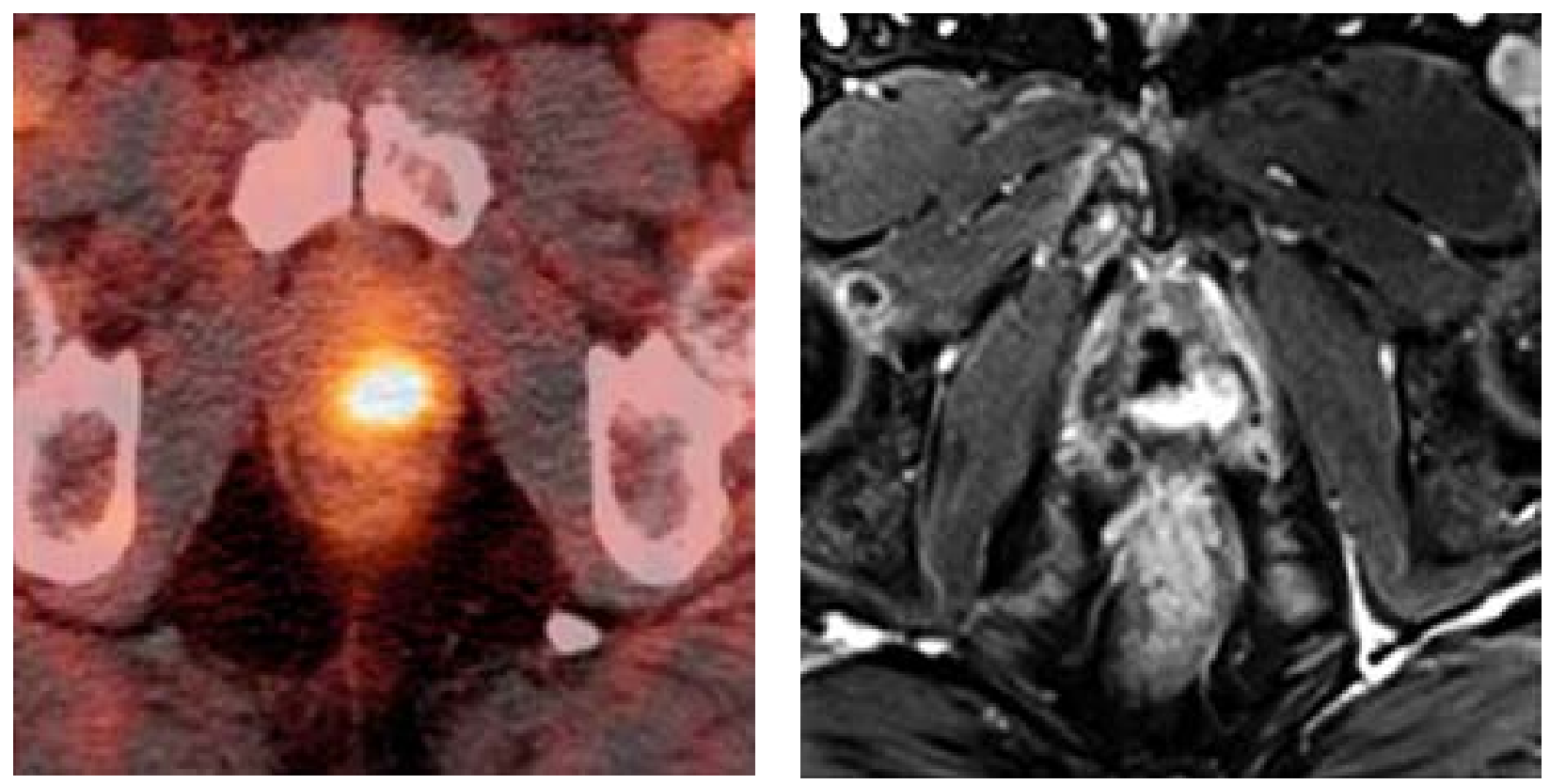

Fig. 4. Axial slices of prostate-specific membrane antigen (PSMA) positron emission tomography combined with computed tomography (PET/CT) (left) and T1w contrast enhanced magnetic resonance imaging (MRI) in patient 2 showing on single lesion of recurrent prostate cancer $(\mathrm{PCa})$

nology may provide additional security in repositioning the BT needles within the biopsy confirmed GTV [43]. For that purpose, a more sophisticated and dedicated image guided biopsy system such BiopSee ${ }^{\circledR}$ (MedCom GmbH, Germany) should be considered. Taking into account all those inaccuracies, we applied a $5 \mathrm{~mm}$ isotropic margin to extract PTV out of GTV.

Huang et al. proved that a significant portion of recurrent PCa after irradiation are distributed in regions of the prostate (apical and periurethral), which are at risk for undertreatment using focal ablative therapies [44]. Our group could show that mpMRI and PSMA PET/CT presented conflicting results for GTV-delineation in patients with primary prostate cancer [28]. Recently, Eiber et al. reported improved localization of primary PCa when imaging was performed with PSMA PET/MRI [45]. Such findings outline the importance of pre-salvage diagnostic imaging and pre-salvage biopsy. Using the combination of molecular (PET) and functional imaging (MRI) followed by guided transperineal biopsy, high PCa detection rates should be achieved. In the case of multimodal confirmed localized PCa, we believe that focal single dose HDR-BT may provide a promising tool in treatment of recurrent PCa after RT. Several studies showed the feasibility of whole gland HDR-BT for recurrent PCa after RT using multiple fractions [46]. However, due to the proximity to rectum and bladder, the opportunity to escalate the treatment doses to an already irradiated prostatic gland is impeded and higher complication rates for salvage BT compared to first line BT have been reported [23]. There is a growing evidence that local control of PCa is dose dependent and increases with hypofractionation $[3,31,47]$. Additionally, multiple fractions of HDR-BT increase the risk of peri-BT complications caused by mechanical irritation during needle and TRUS-probe inser- tion. Thus, the potential advantage of focal HDR-BT is the delivery of a high-dose in a single fraction without increasing the toxicity. Future studies have to address the important question of patient selection. Positively selected patients may have a chance for cure. On the other hand, toxicity rates are higher in salvage therapy compared to primary RT [21]. Furthermore, the role of simultaneous or adjuvant androgen deprivation therapy has to be defined. In current literature, no conclusive data is available $[3,11,22,24]$. Patients with only locally recurrent PCa could harbor a different cancer phenotype. Thus, the benefit of hormones could be less than in patients with locally advanced disease at baseline, which suffer from an increased risk of microscopic occult metastatic PCa [11].

\section{Conclusions}

In this methodology report, we presented a new approach for focal, single dose HDR-BT re-irradiation of locally recurrent PCa. Focal HDR-BT on a multimodal defined GTV may provide a strong tool for a selected subgroup of patients, and may offer a possibility for cure with a low toxicity profile. Further studies have to evaluate the role of this new technique.

\section{Disclosure}

Prof. Philipp Tobias Meyer has received research grants from Company Piramal and GE. The other authors declare no conflicts of interest.

\section{References}

1. Alicikus ZA, Yamada Y, Zhang Z et al. Ten-year outcomes of high-dose, intensity-modulated radiotherapy for localized prostate cancer. Cancer 2011; 117: 1429-1437. 
2. Sylvester JE, Grimm PD, Wong J et al. Fifteen-year biochemical relapse-free survival, cause-specific survival, and overall survival following I (125) prostate brachytherapy in clinically localized prostate cancer: Seattle experience. Int J Radiat Oncol Biol Phys 2011; 81: 376-381.

3. Stone NN, Unger P, Crawford ED et al. Diagnosis and management of local recurrence after low-dose-rate brachytherapy. Brachytherapy 2015; 14: 124-130.

4. Zelefsky MJ, Yamada Y, Fuks Z et al. Long-term results of conformal radiotherapy for prostate cancer: impact of dose escalation on biochemical tumor control and distant metastases-free survival outcomes. Int J Radiat Oncol Biol Phys 2008; 71: 1028-1033.

5. Haider MA, Chung P, Sweet J et al. Dynamic contrast-enhanced magnetic resonance imaging for localization of recurrent prostate cancer after external beam radiotherapy. Int J Radiat Oncol Biol Phys 2008; 70: 425-430.

6. Evangelista L, Zattoni F, Guttilla A et al. Choline PET or PET/CT and biochemical relapse of prostate cancer: a systematic review and meta-analysis. Clin Nucl Med 2013; 38 305-314.

7. Eiber M, Maurer T, Souvatzoglou M et al. Evaluation of Hybrid ${ }^{68}$ Ga-PSMA Ligand PET/CT in 248 Patients with Biochemical Recurrence After Radical Prostatectomy. J Nucl Med 2015; 56: 668-674

8. Dinniwell R, Chan P, Czarnota G et al. Pelvic lymph node topography for radiotherapy treatment planning from ferumoxtran-10 contrast-enhanced magnetic resonance imaging. Int J Radiat Oncol Biol Phys 2009; 74: 844-851.

9. Rud E, Baco E, Lien D et al. Detection of radiorecurrent prostate cancer using diffusion-weighted imaging and targeted biopsies. AJR Am J Roentgenol 2014; 202: 241-246.

10. Kanthabalan A, Emberton M, Ahmed HU. Biopsy strategies for selecting patients for focal therapy for prostate cancer. Curr Opin Urol 2014; 24: 209-217.

11. Créhange G, Roach M 3rd, Martin E et al. Salvage reirradiation for locoregional failure after radiation therapy for prostate cancer: who, when, where and how? Cancer Radiother 2014; 18: 524-534.

12. Agarwal PK, Sadetsky N, Konety BR et al. Treatment failure after primary and salvage therapy for prostate cancer: likelihood, patterns of care, and outcomes. Cancer 2008; 112: 307-314.

13. Freedland SJ, Humphreys EB, Mangold LA et al. Death in patients with recurrent prostate cancer after radical prostatectomy: prostate-specific antigen doubling time subgroups and their associated contributions to all-cause mortality. I Clin Oncol 2007; 25: 1765-1771.

14. Nguyen PL, D'Amico AV, Lee AK et al. Patient selection, cancer control, and complications after salvage local therapy for postradiation prostate-specific antigen failure: a systematic review of the literature. Cancer 2007; 110: 1417-1428.

15. Finley DS, Belldegrun AS. Salvage cryotherapy for radiation-recurrent prostate cancer: outcomes and complications. Curr Urol Rep 2011; 12: 209-215.

16. Leibovici D, Spiess PE, Heller L et al. Salvage surgery for locally recurrent prostate cancer after radiation therapy: tricks of the trade. Urol Oncol 2008; 26: 9-16.

17. Lukka H, Waldron T, Chin J et al. High-intensity focused ultrasound for prostate cancer: a systematic review. Clin Oncol (R Coll Radiol) 2011; 23: 117-127.

18. Jereczek-Fossa BA, Beltramo G, Fariselli L et al. Robotic image-guided stereotactic radiotherapy, for isolated recurrent primary, lymph node or metastatic prostate cancer. Int J Radiat Oncol Biol Phys 2012; 82: 889-897.

19. Chen CP, Weinberg V, Shinohara K et al. Salvage HDR brachytherapy for recurrent prostate cancer after previous de- finitive radiation therapy: 5-year outcomes. Int J Radiat Oncol Biol Phys 2013; 86: 324-329.

20. Beyer DC. Brachytherapy for recurrent prostate cancer after radiation therapy. Semin Radiat Oncol 2003; 13: 158-165.

21. Yamada Y, Kollmeier MA, Pei X et al. A Phase II study of salvage high-dose-rate brachytherapy for the treatment of locally recurrent prostate cancer after definitive external beam radiotherapy. Brachytherapy 2014; 13: 111-116.

22. Yamada Y, Okihara K, Iwata T et al. Salvage brachytherapy for locally recurrent prostate cancer after external beam radiotherapy. Asian J Androl 2015; 17: 899-903.

23. Machtens S, Baumann R, Hagemann J et al. Long-term results of interstitial brachytherapy (LDR-Brachytherapy) in the treatment of patients with prostate cancer. World J Urol 2006; 24: 289-295.

24. Peters M, Maenhout M, van der Voort van Zyp JR et al. Focal salvage iodine-125 brachytherapy for prostate cancer recurrences after primary radiotherapy: a retrospective study regarding toxicity, biochemical outcome and quality of life. Radiother Oncol 2014; 112: 77-82.

25. Sasaki H, Kido M, Miki K et al. Salvage partial brachytherapy for prostate cancer recurrence after primary brachytherapy. Int J Urol 2014; 21: 572-577.

26. Roach M 3rd, Hanks G, Thames H Jr. et al. Defining biochemical failure following radiotherapy with or without hormonal therapy in men with clinically localized prostate cancer: recommendations of the RTOG-ASTRO Phoenix Consensus Conference. Int J Radiat Oncol Biol Phys 2006; 65: 965-974.

27. Eder M, Neels O, Müller M et al. Novel Preclinical and Radiopharmaceutical Aspects of $\left[{ }^{68} \mathrm{Ga}\right]$ Ga-PSMA-HBED-CC: A New PET Tracer for Imaging of Prostate Cancer. Pharmaceuticals (Basel) 2014; 7: 779-796.

28. Zamboglou C, Wieser G, Hennies $S$ et al. MRI versus (68) Ga-PSMA PET/CT for gross tumour volume delineation in radiation treatment planning of primary prostate cancer. Eur J Nucl Med Mol Imaging 2015; 43: 889-897.

29. Zamboglou N, Tselis N, Baltas D et al. High-dose-rate interstitial brachytherapy as monotherapy for clinically localized prostate cancer: treatment evolution and mature results. Int J Radiat Oncol Biol Phys 2013; 85: 672-678.

30. Prada PJ, Jimenez I, González-Suárez H et al. High-dose-rate interstitial brachytherapy as monotherapy in one fraction and transperineal hyaluronic acid injection into the perirectal fat for the treatment of favorable stage prostate cancer: treatment description and preliminary results. Brachytherapy 2012; 11: 105-110.

31. Mavroidis P, Milickovic N, Cruz WF et al. Comparison of different fractionation schedules toward a single fraction in high-dose-rate brachytherapy as monotherapy for low-risk prostate cancer using 3-dimensional radiobiological models. Int J Radiat Oncol Biol Phys 2014; 88: 216-223.

32. Bentzen SM, Dörr W, Gahbauer R et al. Bioeffect modeling and equieffective dose concepts in radiation oncology-terminology, quantities and units. Radiother Oncol 2012; 105: 266268.

33. Nath R, Bice WS, Butler WM et al. AAPM recommendations on dose prescription and reporting methods for permanent interstitial brachytherapy for prostate cancer: report of Task Group 137. Med Phys 2009; 36: 5310-5322.

34. Grosu AL, Weirich G, Wendl C et al. 11C-Choline PET/pathology image coregistration in primary localized prostate cancer. Eur J Nucl Med Mol Imaging 2014; 41: 2242-2248.

35. Afshar-Oromieh A, Zechmann CM, Malcher A et al. Comparison of PET imaging with a (68)Ga-labelled PSMA ligand and (18)F-choline-based PET/CT for the diagnosis of recurrent prostate cancer. Eur J Nucl Med Mol Imaging 2014; 41: 11-20. 
36. Ceci F, Uprimny C, Nilica B et al. (68)Ga-PSMA PET/CT for restaging recurrent prostate cancer: which factors are associated with PET/CT detection rate? Eur J Nucl Med Mol Imaging 2015; 42: 1284-1294.

37. Roeske JC, Forman JD, Mesina CF et al. Evaluation of changes in the size and location of the prostate, seminal vesicles, bladder, and rectum during a course of external beam radiation therapy. Int J Radiat Oncol Biol Phys 1995; 33: 1321-1329.

38. Debois M, Oyen R, Maes F et al. The contribution of magnetic resonance imaging to the three-dimensional treatment planning of localized prostate cancer. Int J Radiat Oncol Biol Phys 1999; 45: 857-865.

39. Smith WL, Lewis C, Bauman G et al. Prostate volume contouring: a 3D analysis of segmentation using 3DTRUS, CT, and MR. Int J Radiat Oncol Biol Phys 2007; 67: 1238-1247.

40. Afshar-Oromieh A, Haberkorn U, Schlemmer HP et al. Comparison of PET/CT and PET/MRI hybrid systems using a 68Ga-labelled PSMA ligand for the diagnosis of recurrent prostate cancer: initial experience. Eur J Nucl Med Mol Imaging 2014; 41: 887-897.

41. Radtke JP, Kuru TH, Boxler S et al. Comparative analysis of transperineal template saturation prostate biopsy versus magnetic resonance imaging targeted biopsy with magnetic resonance imaging-ultrasound fusion guidance. J Urol 2015; 193: 87-94.

42. Ukimura O, Hirahara N, Fujihara A et al. Technique for a hybrid system of real-time transrectal ultrasound with preoperative magnetic resonance imaging in the guidance of targeted prostate biopsy. Int J Urol 2010; 17: 890-893.

43. Ukimura O, Gross ME, de Castro Abreu AL et al. A novel technique using three-dimensionally documented biopsy mapping allows precise re-visiting of prostate cancer foci with serial surveillance of cell cycle progression gene panel. Prostate 2015; 75: 863-871.

44. Huang WC, Kuroiwa K, Serio AM et al. The anatomical and pathological characteristics of irradiated prostate cancers may influence the oncological efficacy of salvage ablative therapies. J Urol 2007; 177: 1324-1329.

45. Eiber M, Weirich G, Holzapfel K et al. Simultaneous Ga-PSMA HBED-CC PET/MRI Improves the Localization of Primary Prostate Cancer. Eur Urol 2016; doi: 10.1016/j.eururo. 2015.12.053 [In press].

46. Hoskin PJ, Colombo A, Henry A et al. GEC/ESTRO recommendations on high dose rate afterloading brachytherapy for localised prostate cancer: an update. Radiother Oncol 2013; 107: 325-332.

47. Martínez-Monge R, Cambeiro M, Ramos LI et al. Volume of high-dose regions and likelihood of locoregional control after perioperative high-dose-rate brachytherapy: do hotter implants work better? Brachytherapy 2014; 13: 591-596. 